\title{
Patent Foramen Ovale as Possible Cause of Sudden Sensorineural Hearing Loss: A Case Report
}

\author{
Andrea Ciorba $^{a}$ Virginia Corazzi $^{\mathrm{a}} \quad$ Luca Cerritelli $^{\mathrm{a}} \quad$ Chiara Bianchini $^{\mathrm{a}}$ \\ Giovanni Scanelli $^{\mathrm{b}}$ Claudia Aimoni $^{\mathrm{a}}$ \\ ${ }^{\mathrm{a}}$ Department of Ear Nose and Throat and Audiology, University Hospital of Ferrara, Ferrara, and ${ }^{\mathrm{b}}$ Internal Medicine, \\ Azienda Unità Locale Socio Sanitaria (AULSS) 8 Berica, San Bortolo Hospital, Vicenza, Italy
}

\section{Significance of the Study}

- This present case showed evidence for the possible role of the patent foramen ovale and, particularly, paradoxical thromboembolism in the pathogenesis of sudden sensorineural hearing loss (SSNHL). Hence, otolaryngologists, audiologists, and internists should consider this condition among the different possible mechanisms involved in the pathogenesis of SSNHL.

\section{Keywords}

Sudden sensorineural hearing loss · Patent foramen ovale . Etiology

\begin{abstract}
Objective: To present a case of sudden sensorineural hearing loss (SSNHL) and the related diagnostic workup. Clinical Presentation and Intervention: A 54-year-old man presented with a unilateral SSNHL episode and vertigo. A severe patent foramen ovale (PFO) that included a complete ear nose and throat, audiological, and neurological examination was discovered during the diagnostic workup. Audiometry, blood, and serological tests were performed. Magnetic resonance imaging and color Doppler echography of the supraaortic trunks and lower limbs were carried out. Finally, transthoracic followed by transesophageal echocardiography
\end{abstract}

\section{KARGER}

E-Mail karger@karger.com www.karger.com/mpp
(C) 2017 The Author(s)

Published by S. Karger AG, Basel

Karger

open access

This is an Open Access article licensed under the Creative Common Attribution-NonCommercial-4.0 International License (CC BY-NC) (http://www.karger.com/Services/OpenAccessLicense), applicable to the online version of the article only. Usage and distribution for com mercial purposes requires written permission. and transcranial Doppler were performed in order to confirm the diagnosis of PFO. Medical therapy with systemic steroid and antiplatelet drugs was administered. Conclusions: This case showed a small PFO associated with an aneurysmatic interatrial septum. The PFO and paradoxical thromboembolism could be involved in the pathogenesis of SSNHL.

(c) 2017 The Author(s)

Published by S. Karger AG, Basel

\section{Introduction}

Sudden sensorineural hearing loss (SSNHL) is described as a sensorineural (generally unilateral) hearing defect of at least $30 \mathrm{~dB}$ for at least 3 contiguous frequencies with an abrupt onset (within 3 days) $[1,2]$. The SSNHL incidence is reported to be 5-20 per 100,000 , to

Andrea Ciorba, MD, PhD

Department of Ear Nose and Throat and Audiology University Hospital of Ferrara (loc. Cona), Via A. Moro 8

IT-44124 Ferrara (Italy)

E-Mail andrea.ciorba@unife.it 
occur independently of sex, and mainly between the fifth and the sixth decade of life $[1,2]$.

In most cases, the etiology remains unknown, and, as a consequence, SSNHL is classified as idiopathic. Several theories about the pathophysiology of idiopathic SSNHL have been suggested, such as vascular and infective [1-3]; unfortunately, only in few cases is it possible to define a causative agent. A possible cause of SSNHL [4] could be a patent foramen ovale (PFO), as it is a frequent embryological residual of fetal foramen ovale, consisting of a virtual interatrial communication which may permit an anomalous blood flow from the right atrium to the left one whenever a Valsalva maneuver is performed [1-5]. The PFO is a common finding in the general population and may be completely asymptomatic [5]. Rarely, patients with PFO may complain of migraine or migrainelike symptoms [5]. Nonetheless, PFO has been associated with an increased risk of cryptogenic ischemic stroke and even more in cases of association with atrial septal aneurysm [1-5]. The supposed pathogenic mechanism is a paradoxical thromboembolism from the venous system to the systemic circulation through the PFO; in addition, a small embolus (1-2 $\mathrm{mm}$ ) may determine an acute cerebral ischemic event [1-5]. Therefore, the presence of PFO and paradoxical thromboembolism could be considered among vascular causes of SSNHL [1-5]. The same mechanism could be advocated in order to explain the onset of SSNHL in particular cases. Hence, the aim of this case report was to describe a case of SSNHL as a possible consequence of a PFO and paradoxical thromboembolism.

\section{Case Report}

A 54-year-old man was referred to our department due to the onset of sudden hearing loss in the right ear and vertigo since the morning. His clinical history was normal - in particular, there was no history of cardiovascular diseases; he also said that he performed sporting activity on a regular basis (running 2 times a week). The ear, nose, and throat examination was unremarkable, except for the presence of a left-beating spontaneous horizontal nystagmus. Pure tone audiogram showed severe pantonal sensorineural hearing loss on the right and normal hearing on the left; tympanogram was type A bilaterally. The neurological evaluation was negative. Systemic steroid therapy was administrated immediately (intramuscular prednisone $1 \mathrm{mg} / \mathrm{kg} /$ day) for 10 days.

Blood tests revealed hypercholesterolemia (total cholesterol $=$ $227 \mathrm{mg} / \mathrm{dL}$; low-density lipoprotein = $166 \mathrm{mg} / \mathrm{dL}$; high-density lipoprotein $=55 \mathrm{mg} / \mathrm{dL})$ and hyperhomocisteinemia (16.7 $\mu \mathrm{mol} / \mathrm{L}$ ), previously unknown, and microcytosis (hemoglobin $=$ $13.8 \mathrm{~g} / \mathrm{dL}$; mean corpuscular volume $=63 \mathrm{fL}$; mean corpuscular hemoglobin $=20.8 \mathrm{pg}$ ), a common condition among the popula-

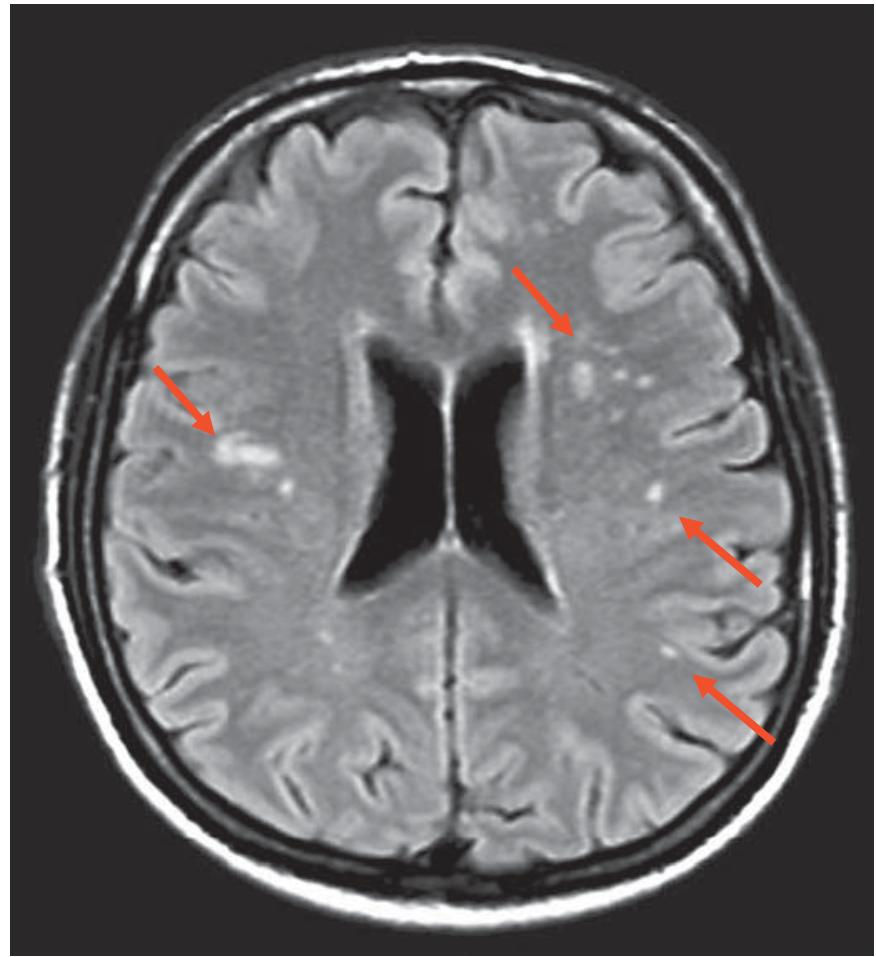

Fig. 1. Cerebral MRI, axial section: multiple T2 hyperintense spots in supratentorial, periventricular, and subcortical regions (arrows), revealing a previously unknown chronic cerebral ischemic disease.

tion of this geographic area (delta of the Po river). No alterations of the clotting/coagulation profile were found, and the thrombophilic screening excluded the principal hereditary thrombophilic disorders. The serologic evaluation excluded the presence of acute viral infections (EBV, herpes varicella zoster, CMV) and autoimmune diseases (Antinuclear antibodies, extractable nuclear antigens, lupus anticoagulant and anticardiolipin antibodies). No electrocardiographic alterations were found while the patient recovered.

Cerebral magnetic resonance imaging (MRI) excluded retrocochlear pathologies, but highlighted multiple T2 hyperintense spots in supratentorial, periventricular, and subcortical regions. Though aspecific, this evidence was considered to be a previously unknown chronic cerebral ischemic disease (Fig. 1). The intracranial vascularization of the MRI study disclosed a thin right vertebral artery and the absence of the left posterior communicating artery (Fig. 2).

To exclude possible origins of thromboembolism, color Doppler echography of the supra-aortic trunks and lower limbs was performed, with no evidence of atherosclerotic plaques and deep venous thrombosis. A successive transthoracic echocardiography revealed an aneurysmatic interatrial sept, with an excursion of 1.9 $\mathrm{cm}$, but could not exclude a cardiac endocavitary thrombus. The following transesophageal echocardiography did not reveal any endocavitary thrombosis and confirmed the interatrial defect, verifying its opening during a Valsalva maneuver (right to left tran- 


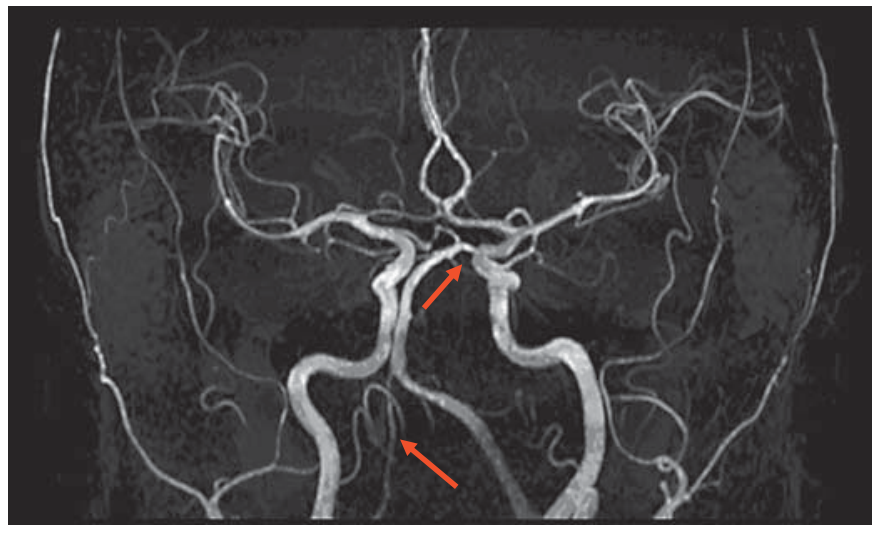

Fig. 2. Intracranial vascularization in MRI study: thin right vertebral artery and absence of the left posterior communicating artery (arrows).

septal passage of microbubbles). A PFO of about $0.5 \mathrm{~mm}$ was diagnosed by the cardiologist (D. Mele). Furthermore, contrast inoculation at the transcranial Doppler highlighted numerous (about 9-10) high-intensity transient signals at rest and a "curtain" effect after the Valsalva maneuver, and the neurologist diagnosed a permanent right-to-left cardiopulmonary shunt. Consequently, the patient was treated with antiplatelet therapy (clopidogrel $75 \mathrm{mg} /$ day); considering the clinical characteristics, the cardiologist gave no indication of PFO surgical treatment.

After 18 months of follow-up, no hearing improvement was noticed in the audiograms. The patient agreed to the publication of the clinical data.

\section{Discussion}

This case showed a small PFO (about $0.5 \mathrm{~mm}$ ) associated with an aneurysmatic interatrial septum. A plausible physiopathological mechanism involved in the onset of SSNHL could be represented by the paradoxical thromboembolism through the PFO, because of the numerous Valsalva maneuvers to which the patient was exposed during regular sporting activity as revealed in the cerebral MRI. However, the angio-MRI showed a thin right vertebral artery and absence of the left posterior communicating artery, which had not been reported to be directly linked to the onset of SSNHL [6-10], and neither influence the hypothesized thromboembolism mechanism nor represent a potential defect of the posterior circulation [10]. Furthermore, some characteristics of the patient disease are known factors associated with an increased risk of stroke occurrence in cases of PFO [8]. In particular, the right-to-left shunt, the

Patent Foramen Ovale and Sudden

Sensorineural Hearing Loss prothrombotic state sustained by the hypercholesterolemia and hyperhomocysteinemia (the latter is also reported to be considered as a cardiovascular risk factor), and the chronic cerebral ischemic disease [8] should allow consideration for the paradoxical thromboembolism to be responsible for the onset of SSNHL in the presented case. Despite the punctual administration of a high dosage of steroids and antiaggregant therapy, the patient did not experience any functional (hearing) improvement; this may also be linked to irreversible vascular damage of inner ear neuroepithelium of possible microvascular origin. To date, we could not retrieve official guidelines concerning PFO treatment. However, it has been reported that no treatment is recommended in asymptomatic cases, while antiplatelet and/or anticoagulant therapy is suggested in some others [8]. When indicated, depending on the clinical characteristics (i.e., age, risk factors for stroke, size of PFO, coexistence of atrial septal aneurysm, tolerance to antiplatelet and anticoagulant drugs) and, in particular, in cases of recurrent ischemic events despite the medical treatment, PFO can be surgically managed and its closure achieved through percutaneous devices [8]. Systemic steroid therapy and antiplatelet drugs were administrated in the present case, according to the most accepted medical treatment suggested for SSNHL and PFO, respectively $[1,8]$.

\section{Conclusions}

This case showed a small PFO associated with an aneurysmatic interatrial septum. PFO and paradoxical thromboembolism could be considered as a possible pathophysiological mechanism of SSNHL.

\section{Acknowledgment}

The authors would like to thank Donato Mele, MD, the cardiologist at the University Hospital of Ferrara, for technical assistance and expertise in the diagnosis of PFO.
Med Princ Pract 2017;26:491-494 DOI: $10.1159 / 000484247$ 


\section{References}

1 Schreiber BE, Agrup C, Haskard DO, et al: Sudden sensorineural hearing loss. Lancet 2010;375:1203-1211.

2 Aimoni C, Bianchini C, Borin M, et al: Diabetes, cardiovascular risk factors and idiopathic sudden sensorineural hearing loss: a casecontrol study. Audiol Neurootol 2010;15: 111-115.

3 Ezerarslan H, Çandar T, Özdemir S, et al: Plasma glycated albumin levels clearly detect hearing loss and atherosclerosis in patients with impaired fasting glucose. Med Princ Pract 2016;25:309-315.
4 Homma S, Di Tullio MR: Patent foramen ovale and stroke. J Cardiol 2010;56:134-141.

5 Mas JL, Arquizan C, Lamy C, et al: Patent foramen ovale and atrial septal aneurysm study group. Recurrent cerebrovascular events associated with patent foramen ovale, atrial septal aneurysm, or both. N Engl J Med 2001;345: 1740-1746.

6 Merchant SN, Durand ML, Adams JC: Sudden deafness: is it viral? ORL J Otorhinolaryngol Relat Spec 2008;70:52-60; discussion 60-62.

7 Iguchi Y, Kimura K, Kobayashi K, et al: Sudden deafness and right-to-left shunts. Cerebrovasc Dis 2008;26:409-412.
8 Meier B, Lock JE: Contemporary management of patent foramen ovale. Circulation 2003;107:5-9.

9 Ciorba A, Faita A, Bianchini C, et al: Arteriopathy and microvascular impairment in sudden sensorineural hearing loss: clues from two clinical cases. Hearing Balance Commun 2013;11:87-90.

$10 \mathrm{Kim}$ C, Sohn JH, Jang MU, et al: Ischemia as a potential etiologic factor in idiopathic unilateral sudden sensorineural hearing loss: analysis of posterior circulation arteries. Hear Res 2016;331:144-151. 